\title{
Gestión de recursos humanos y ética en servidores municipales
}

Mg. Joanna Rodríguez García

jrg015@hotmail.com

Escuela de posgrado

Universidad César Vallejo

ORCID: 0000-0003-2178-3326

\section{Dr. José Manuel Delgado Bardales jmdelgadob@ucvvirtual.edu.pe \\ Escuela de posgrado \\ Universidad César Vallejo ORCID: 0000-0001-6574-2759 \\ Scopus autor ID: 24070333700 \\ Código Renacyt: P0050554}

\section{RESUMEN}

El estudio planteó diseñar una propuesta de gestión de recursos humanos que contribuya éticamente a la prestación de servicios en la Municipalidad Provincial de Rioja. Investigación básica, de diseño descriptivo propositivo. Cabe precisar que el estudio tiene como finalidad generar una propuesta, iniciando coordinaciones con la municipalidad, adicionando un vínculo con los ciudadanos. Posterior a ello, aplicación de instrumentos de recojo de datos. Asimismo, se podrá interactuar con el entrevistado por medio telefónico y recabar los elementos necesarios para el estudio. Se revisaron (20) artículos relevantes y de gran impacto. Concluyendo que la prestación de servicios debe tener características fiables para los ciudadanos, donde primero debe cerrar brechas, ampliando la cobertura de servicios, agregando calidad y cantidad de servicio; en busca de generar valor público que sea capaz de satisfacer las necesidades y expectativas de los ciudadanos.

De la misma manera, los investigadores precisan que es pertinente mejorar las condiciones institucionales, principalmente la estructura y funcionalidad, que son elementos fundamentales para la reestructuración organizacional, donde facilitara el acercamiento a la ciudadanía.

Palabras clave: Gestión de recursos, recursos humanos, gobierno municipal, ética profesional. 


\title{
Management of human resources and ethics in municipal servers
}

\begin{abstract}
The study proposed designing a human resource management proposal that ethically contributes to the provision of services in the Provincial Municipality of Rioja. Basic research with a descriptive purposeful design. It should be noted that the purpose of the study is to generate a proposal, initiating coordination with the municipality, adding a link with citizens. After that, application of data collection instruments. Likewise, it is possible to interact with the interviewee by telephone and collect the necessary elements for the study. (20) relevant and high-impact articles were reviewed. Concluding that the provision of services must have reliable characteristics for citizens, where it must first close gaps, expanding the coverage of services, adding quality and quantity of service; in search of generating public value that is capable of satisfying the needs and expectations of citizens. In the same way, the researchers specify that it is pertinent to improve institutional conditions, mainly the structure and functionality, which are fundamental elements for organizational restructuring, where it will facilitate the approach to citizenship.
\end{abstract}

Keywords: Resource management, human resources, municipal government, professional ethics.

Artículo recibido: 03 nov. 2020 Aceptado para publicación: 07 dic. 2020 Correspondencia:jrg_015@hotmail.com. Conflictos de Interés: Ninguna que declarar 


\section{INTRODUCCIÓN}

Los gobiernos municipales confieren diversas atribuciones sustentadas en la parte normativa para gobernaran democráticamente y con las cuales se estimó que se identificaban los componentes fundamentales para superar las condiciones de los rezagos estructurales de los municipios, en los albores del siglo XXI resultan insuficientes para conseguir los cambios en curso que culminen en mejores niveles de bienestar, desarrollo y progreso en el ámbito territorial de los municipios. La magnitud de los problemas políticos, jurídicos, administrativos, económicos y sociales municipales se ha intensificado en los últimos tiempos, y lejos de que los gobiernos municipales, al gobernar, disminuyan la brecha entre los contrastes sociales, tales como desigualdad, pobreza, marginación, desempleo, inseguridad, entre otros aspectos, se han incrementado (Olivos, 2013).

En ese sentido los gobiernos deben responder a las demandas, teniendo entre sus actividades administrativas ofrecer los servicios públicos de agua potable, drenaje, alcantarillado, tratamiento y disposición de aguas residuales, alumbrado público, limpia, recolección, traslado, tratamiento y disposición final de residuos urbanos, hacienda pública, gobernación y reglamentos, seguridad pública, tránsito y transportes, obras públicas, instrucción pública, recreación y espectáculos, comercios, mercados y rastro, bienes municipales, panteones, y los demás que las legislaturas locales determinen (Instituto Nacional de Administración Pública A.C. (INAP), 2009). En el sector público, la necesidad de comenzar el proceso de reforma del Estado se sitúa como lineamiento estratégico central para comprender las posibilidades de éxito o fracaso de estos objetivos estratégicos. Donde la modernización del sector productivo y la adecuación de los servicios a las necesidades de la ciudadanía se constituyen en aspectos clave para el desarrollo económico y social del país (Supervielle \& Pucci, 2015).

Por otro lado, es indudable la importancia que adquirió la gestión de los recursos humanos en los últimos años en las organizaciones públicas. Esta tendencia puso en valor el papel de las personas para el logro de los fines institucionales y la necesidad de encontrar políticas, estrategias y mecanismos para mejorar la gestión de los recursos humanos adscriptos a los aparatos administrativos del estado. En este marco, la concepción sobre los recursos humanos en la gestión local, adquiere un relieve singular. Los diferentes enfoques respecto del desarrollo local no pasan por alto la importancia de los recursos humanos como un elemento estratégico de dicho desarrollo. En efecto, las personas dejaron de entenderse 
como parte del capital físico de una organización (factor humano), como un "recurso", para entenderlas como agentes de cambio que poseen un capital intangible de valor irremplazable: el conocimiento. Precisamente, el conocimiento, junto a la imaginación humana, es y será la fuente principal de riqueza de una sociedad (Bonardo, 2009).

Por esta razón, las organizaciones necesitan más que nunca considerar cuidadosamente la gestión de recursos humanos con el fin de incrementar su capacidad para atraer, desarrollar y retener a los trabajadores más valiosos (Cappelli, 2000). Si bien todos los empleados poseen competencias que potencialmente pueden crear valor para la organización, existen individuos que se diferencian de sus pares por su capacidad para producir una contribución aún mayor (Aguinis \& O'Boyle, 2014). La identificación de estas contribuciones diferenciales y la comprensión acerca de cómo gestionar mejor a los empleados más valiosos resulta fundamental para la construcción de nuevas capacidades competitivas. En ese sentido, el capital humano constituye la base esencial para el desarrollo de ventajas competitivas sostenibles en la organización (Becker, Huselid, \& Beatty, 2009)

Por tanto, la función de Recursos Humanos ha sufrido una gran evolución en las últimas décadas. En consecuencia, se han producido cambios sustanciales en su rol, cada vez más estratégico, y en su contenido, que han supuesto una ampliación de las funciones a abordar y una sofisticación de las prácticas utilizadas. Dicha evolución articula implicaciones a nivel organizativo y estratégico, por lo que se destaca la necesidad de relacionar las políticas y los sistemas de prácticas de RRHH con los objetivos estratégicos de la empresa (Delery \& Doty, 1996). Convirtiéndose en elemento fundamental para la ventaja competitiva y por lo tanto éste se constituye en un componente esencial para cualquier tipo de institución. Este elemento común es el gran diferenciador que hace que haya competitividad puesto que esta debe demostrarse, debe medirse y se debe comparar (Gónzalez, 2005). No cabe duda que en la organización quien logra la gestión y el cumplimiento tanto de las metas como de los objetivos establecidos, es el recurso humano y que ellos son el factor estratégico de la organización (Ortíz, Rendón, \& Atehortúa, 2012) Donde el afán del gobierno local es generar una prestación de los servicios de manera oportuna, donde es necesario el uso de las Tecnologías de la Información y Comunicación (TICs) se ha vuelto una estrategia común para el desarrollo y soporte de la relación entre los ciudadanos y el gobierno. Particularmente, los gobiernos locales, referidos a comunidades más acotadas, las que normalmente corresponden a municipios (Sá, Rocha, 
\& Pérez, 2016). Éstos, se han convertido durante los últimos años en proveedores de servicios muy variados, en los que el uso de sistemas online posibilita, a diferencia de los mecanismos tradicionales, un servicio continuo a lo largo de todo el día y de amplio alcance (Chaín, Muñoz, \& Más, 2008). Es así que en el ámbito local, el gobierno electrónico (egovernment) adquiere su máxima dimensión y relevancia, dado que los municipios son las organizaciones públicas más cercanas a los ciudadanos y les proporcionan directamente más servicios que ninguna otra administración (Sandoval-Almazan \& Mendoza, 2011).

\section{MATERIALES Y MÉTODOS}

Investigación básica, caracteriza por no alterar ningún elemento que se investiga, manteniéndose en su estado natural (Hernández, 2014). El diseño de investigación es descriptivo con revisión sistemática (Hernández, 2016). La técnica de análisis se desarrolló a través de la revisión sistemática evaluando cada uno de los artículos para una comparación de los puntos o características en las cuales concuerda y los puntos en los que existe discrepancia entre artículos nacionales e internacionales. Además, de acuerdo a criterios técnicos pre establecidos, se realizó una evaluación crítica e intensiva de cada artículo, determinando la calidad de la evidencia y la fuerza de recomendación para cada artículo.

Criterios de elegibilidad, según tipos de participantes: se incluyeron estudios que involucraban a adultos y personas mayores servidores en los gobiernos locales, siendo excluidas aquellas revisiones de literatura de naturaleza teórica. Tipos de estudios: Teniendo en cuenta el número limitado de estudios sobre gestión de recursos humanos en los gobiernos locales en Latinoamérica, el objetivo de esta breve reseña es trazar un mapa de los conocimientos existentes sobre el tema e identificar los diseños de estudio por el nivel de las pruebas. Tipos de resultados: Se seleccionaron como resultados primarios los siguientes: casos de estudios, investigaciones empíricas, experiencias y similares.

El proceso de selección de los estudios fue realizado por dos revisores independientes, y cualquier divergencia fue resuelta por un tercer revisor. Los estudios se seleccionaron en dos etapas. El primer paso consistió en revisar los títulos y resúmenes de las referencias encontradas con nuestra estrategia de búsqueda; se seleccionaron los estudios potencialmente elegibles. El segundo paso consistió en revisar el texto completo de los estudios preseleccionados para confirmar su elegibilidad. 


\section{RESULTADOS Y DISCUSIÓN}

3.1. Tablas 1: Estudios revisados sobre servicios municipalidades.

DATOS DE LA PUBLICACION

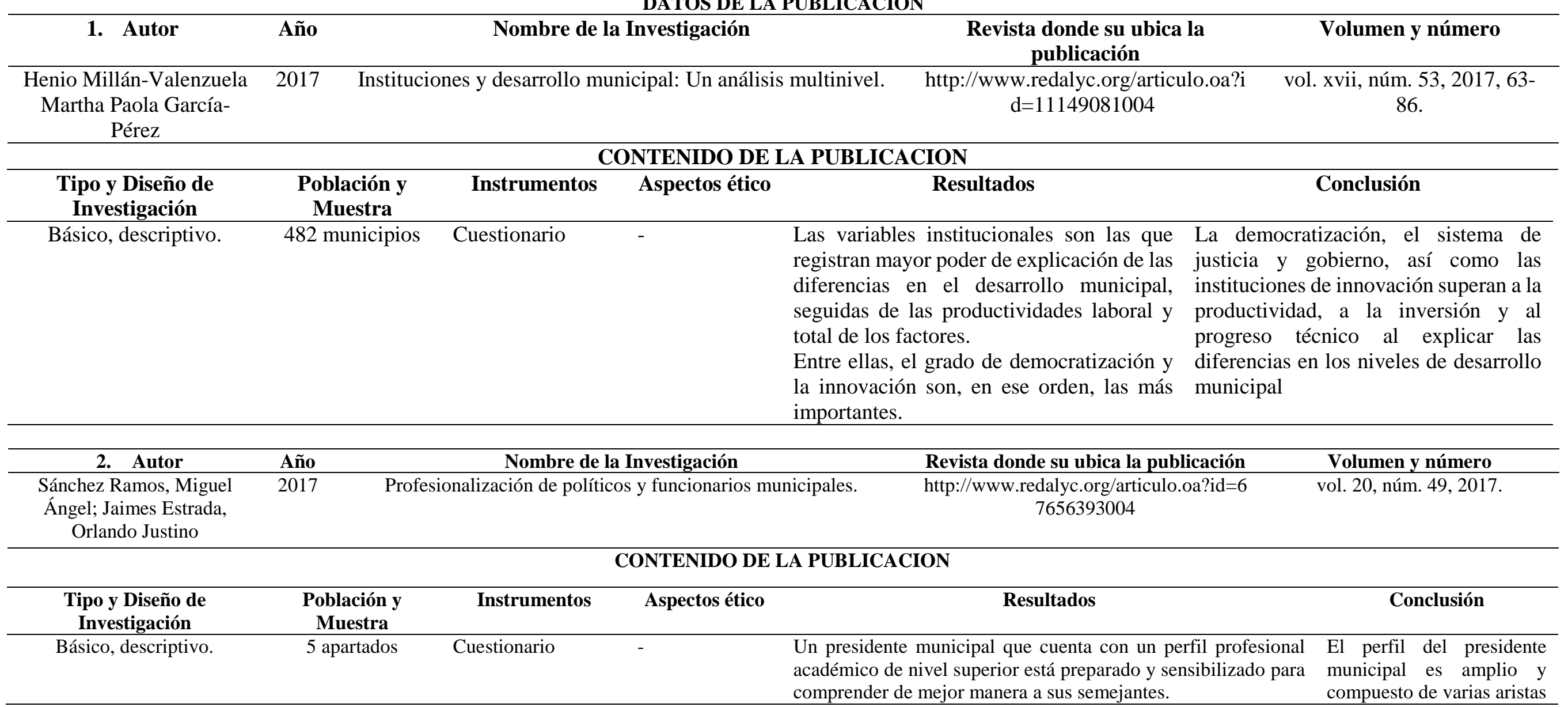


Le facilitará la interpretación de necesidades y tiene en su haber elementos y técnicas para

la conducción del gobierno y la administración. Esto implica facilidad para con el trato y

las relaciones interpersonales, criterio desarrollado, aptitud y actitud de líder no sólo la académica, sino también la política y tal vez esta sea la más determinante para el desempeño en el cargo.

\begin{tabular}{|c|c|c|c|c|c|}
\hline 3. $\quad$ Autor & Año & \multicolumn{2}{|c|}{ Nombre de la Investigación } & Revista donde su ubica la publicación & Volumen y número \\
\hline Hellmut Wollmann & La pr & \multicolumn{3}{|c|}{$\begin{array}{l}\text { La provisión de servicios públicos municipales: ¿un movimiento http:// } \\
\text { pendular? }\end{array}$} & núm. 26, 2019. \\
\hline \multicolumn{6}{|c|}{ CONTENIDO DE LA PUBLICACION } \\
\hline $\begin{array}{l}\text { Tipo y Diseño de } \\
\text { Investigación }\end{array}$ & $\begin{array}{l}\text { Población y } \\
\text { Muestra }\end{array}$ & Instrumentos & Aspectos ético & Resultados & Conclusión \\
\hline Básico, descriptivo. & 5 países & $\begin{array}{l}\text { Cuestionario } \\
\text { Revisión } \\
\text { documental }\end{array}$ & - & $\begin{array}{l}\text { La fase más reciente muestra una "bifurcación" notoria de las } \\
\text { corrientes y tendencias. Por un lado, resulta una corriente } \\
\text { promovida por la política de la Unión Europea que continúa } \\
\text { impulsando la liberalización del mercado y así la contratación y } \\
\text { privatización. Por otro lado, se observa (como una tendencia } \\
\text { paralela y en parte opuesta, un "retorno" (comeback) del sector } \\
\text { municipal y de sus empresas en la prestación de los servicios } \\
\text { públicos (remunicipalización). } \\
\text { Además, en el campo de los servicios sociales personales y de la } \\
\text { ayuda social re-emergen y re-aparecen organizaciones y actores } \\
\text { sociales constituyéndose en representantes clave del tercer } \\
\text { sector, incluyendo actores e iniciativas de la sociedad civil lo que } \\
\text { podría interpretarse como cierta "re-sociedadización". }\end{array}$ & $\begin{array}{l}\text { En esta perspectiva, el re- } \\
\text { surgir de las iniciativas de } \\
\text { la "sociedad civil" podría } \\
\text { llamarse } \\
\text { sociedadización". } \\
\text { embargo, aunque sin } \\
\text { intelectualmente atractivo y } \\
\text { también heurísticamente } \\
\text { útil, hay que darse cuenta } \\
\text { de que existen grandes } \\
\text { diferencias estructurales y } \\
\text { socioeconómicas entre } \\
\text { aquel entonces y la } \\
\text { actualidad. }\end{array}$ \\
\hline
\end{tabular}

\begin{tabular}{|c|c|c|c|c|}
\hline 4. $\quad$ Autor & Año & \begin{tabular}{|c|} 
Nombre de la Investigación \\
\end{tabular} & Revista donde su ubica la publicación & Volumen y número \\
\hline $\begin{array}{l}\text { Miguel López- } \\
\text { Cabanas }\end{array}$ & 2018 & Servicios sociales municipales. Necesidad de una refundación & $\begin{array}{l}\text { http://www.redalyc.org/articulo.oa?id=7 } \\
7855949002\end{array}$ & $\begin{array}{l}\text { vol. 39, núm. 2, } \\
2018\end{array}$ \\
\hline
\end{tabular}

Ciencia Latina Revista Científica Multidisciplinar, Ciudad de México, México. ISSN 2707-2207 / ISSN 2707-2215 (en línea), julio-diciembre, 2020, Volumen 4, Número 2. https://doi.org/10.37811/cl rcm.v4i2.149 p. 1209 


\begin{tabular}{|c|c|c|c|c|c|}
\hline \multicolumn{6}{|c|}{ CONTENIDO DE LA PUBLICACION } \\
\hline $\begin{array}{l}\text { Tipo y Diseño de } \\
\text { Investigación }\end{array}$ & Población y Muestra & Instrumentos & Aspectos ético & Resultados & Conclusión \\
\hline Básico, descriptivo. & 10 ayuntamientos & $\begin{array}{l}\text { Revisión } \\
\text { documental }\end{array}$ & - & $\begin{array}{l}\text { Existe una confusión en cuanto a su misión y su } \\
\text { delimitación con otros ámbitos de las políticas } \\
\text { sociales. Hay una sobrecarga de trabajo burocrático y } \\
\text { administrativo que limita las actuaciones propias de } \\
\text { los profesionales de la Intervención Social. Es } \\
\text { necesario realizar una refundación de los Servicios } \\
\text { Sociales que prestan los ayuntamientos, que debería de } \\
\text { pasar por su reconceptualización e importantes } \\
\text { cambios normativos, organizativos y metodológicos }\end{array}$ & $\begin{array}{l}\text { Existen bastantes argumentos para } \\
\text { coincidir con la afirmación de que } \\
\text { podríamos estar ante la posibilidad de } \\
\text { un colapso estructural, lo que habría } \\
\text { que asumir como una oportunidad } \\
\text { para una reforma de todo el Sistema } \\
\text { de Servicios Sociales. En todo caso, } \\
\text { hay que dejar de hacer una atribución } \\
\text { externa de las responsabilidades y } \\
\text { asumir las propias, que pasan, qué } \\
\text { duda cabe, por un profundo cambio } \\
\text { de paradigma que conduzca a la } \\
\text { ineludible refundación de los } \\
\text { Servicios Sociales municipales. }\end{array}$ \\
\hline
\end{tabular}

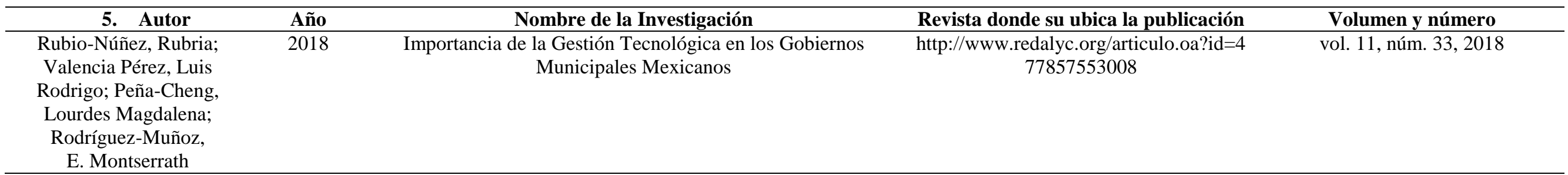

\section{CONTENIDO DE LA PUBLICACION}

\begin{tabular}{|c|c|c|c|c|c|}
\hline $\begin{array}{l}\text { Tipo y Diseño de } \\
\text { Investigación }\end{array}$ & $\begin{array}{c}\text { Población y } \\
\text { Muestra }\end{array}$ & Instrumentos & Aspectos ético & Resultados & Conclusión \\
\hline Básico, aplicativo & 45 municipios & Cuestionario & & $\begin{array}{l}\text { tienen bajo su responsabilidad proporcionar servicios } \\
\text { públicos que garanticen la calidad de vida adecuada } \\
\text { para sus ciudadanos, además deben enfrentar } \\
\text { situaciones como el crecimiento demográfico derivado } \\
\text { de la migración de zonas rurales a las zonas urbanas, } \\
\text { lo que se traduce en hacer esfuerzos mayores para }\end{array}$ & $\begin{array}{l}\text { Para cumplir, es necesario } \\
\text { apoyarse en la identificación e } \\
\text { incorporación de la tecnología, } \\
\text { así como su gestión, lo que } \\
\text { conlleva a que los gobernantes, } \\
\text { ciudadanía, empresas y demás } \\
\text { actores involucrados en los }\end{array}$ \\
\hline
\end{tabular}

Ciencia Latina Revista Científica Multidisciplinar, Ciudad de México, México. ISSN 2707-2207 / ISSN 2707-2215 (en línea), julio-diciembre, 2020, Volumen 4, Número 2. https://doi.org/10.37811/cl_rcm.v4i2.149 p. 1210 
incrementar su infraestructura, plantear y llevar a cabo la planificación urbana y hacer rendir sus recurso municipios, se apropien de una visión enfocada a la gestión tecnológica en el municipio.

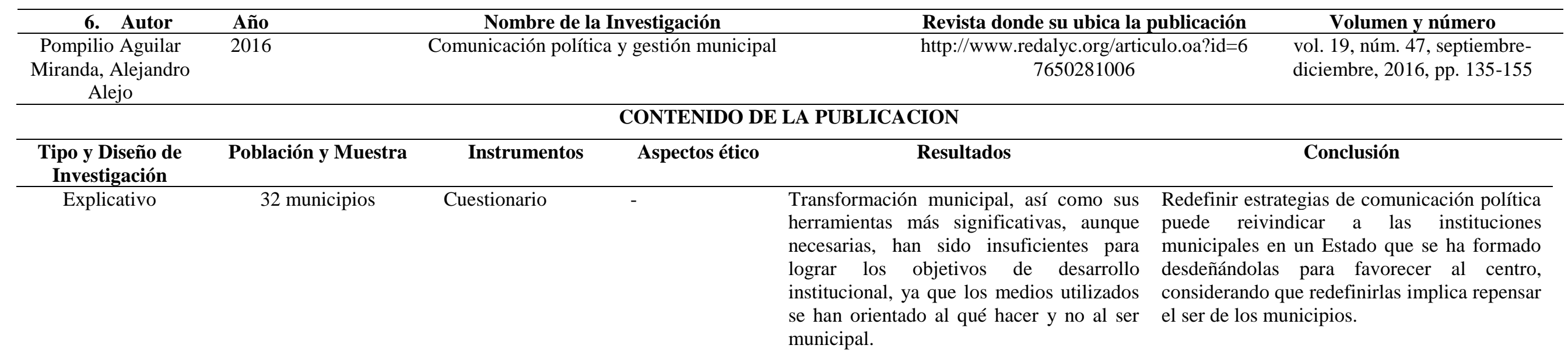

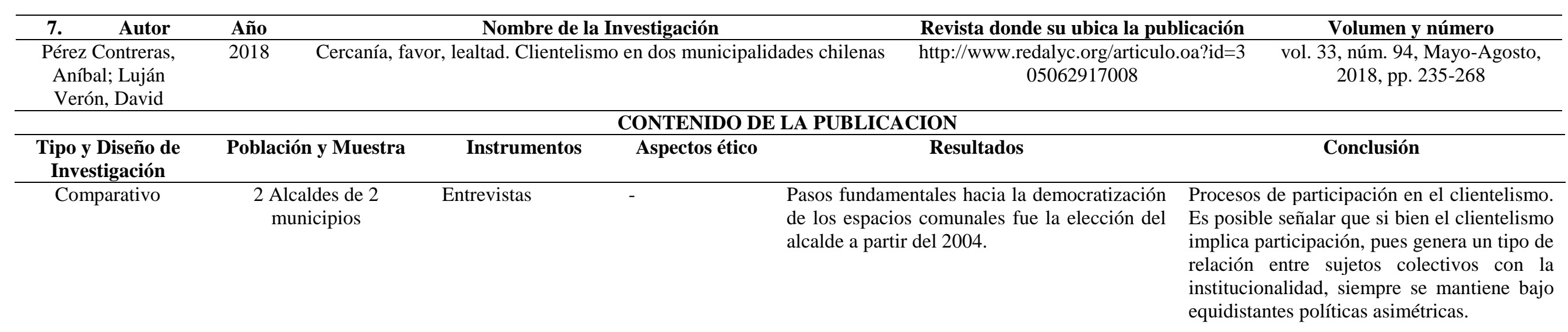

Ciencia Latina Revista Científica Multidisciplinar, Ciudad de México, México. 


\subsection{Tablas 2: Estudios revisados sobre gestión de recursos humanos}

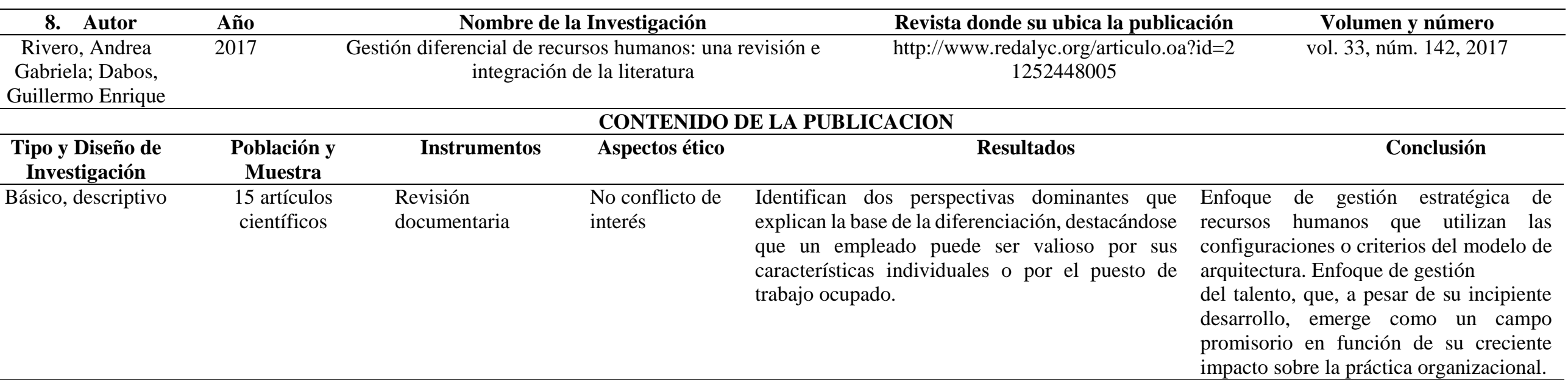

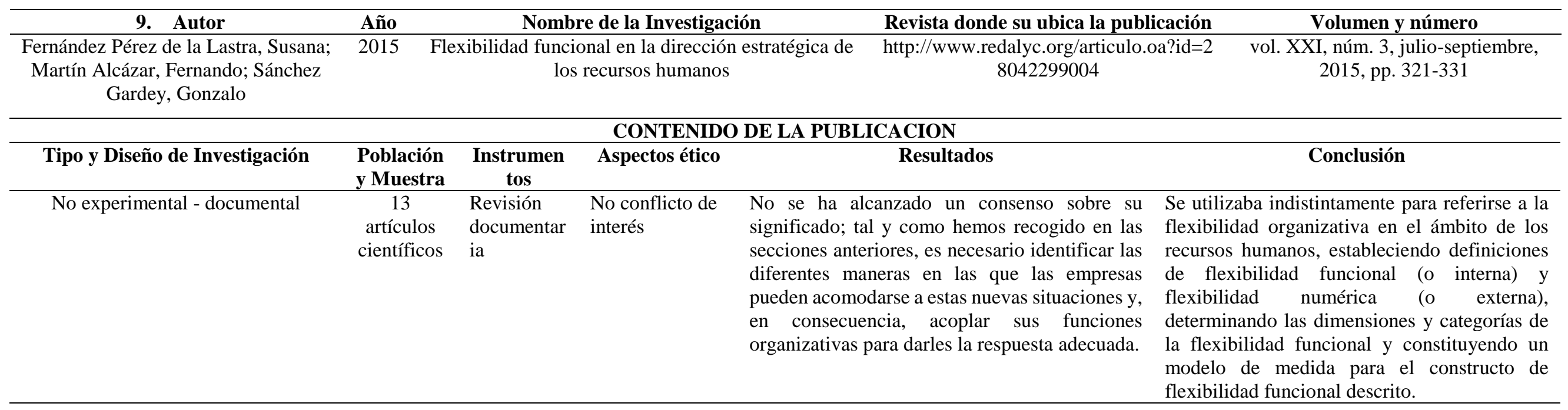

Ciencia Latina Revista Científica Multidisciplinar, Ciudad de México, México. 


\begin{tabular}{|c|c|c|c|c|c|c|c|}
\hline \multicolumn{2}{|l|}{ 10. Autor } & \multicolumn{3}{|c|}{ Nombre de la Investigación } & \multicolumn{2}{|c|}{ Revista donde su ubica la publicación } & Volumen y número \\
\hline $\begin{array}{r}\text { Medina Lorza, Aida; } \\
\text { Taboada, Lorenzo; Aco } \\
\text { Alejandro }\end{array}$ & lito & Perfil de & $\begin{array}{l}\text { el responsable y su } \\
\text { de Recurso }\end{array}$ & $\begin{array}{l}\text { mpacto en la arquitectura } \\
\text { Humanos }\end{array}$ & $\begin{array}{r}\text { http://www.redalyc.org } \\
90514570\end{array}$ & $\begin{array}{l}\text { articulo.oa?id=2 } \\
066\end{array}$ & $\begin{array}{l}\text { vol. 22, núm. 77, enero-marzo, } \\
\text { 2017, pp. 75-94 }\end{array}$ \\
\hline \multicolumn{8}{|c|}{$\begin{array}{l}\text { CONTENIDO DE LA PUBLICACION } \\
\end{array}$} \\
\hline $\begin{array}{l}\text { Tipo y Diseño de } \\
\text { Investigación }\end{array}$ & $\begin{array}{l}\text { Población } \\
\text { y Muestra }\end{array}$ & $\begin{array}{l}\text { Instrumen } \\
\text { tos }\end{array}$ & Aspectos ético & Resul & dos & & Conclusión \\
\hline Básico, descriptivo & $\begin{array}{c}600 \\
\text { personas } \\
\text { responsabl } \\
\text { es de } \\
\text { RRHH }\end{array}$ & $\begin{array}{l}\text { Cuestionari } \\
\text { o }\end{array}$ & $\begin{array}{l}\text { No conflicto de } \\
\text { interés }\end{array}$ & $\begin{array}{l}\text { El nivel de competencia } \\
\text { no parece influir significa } \\
\text { ajuste del sistema de p } \\
\text { empresa a la arquitectura } \\
\text { Snell (1999). Es decir, } \\
\text { competente el responsabl } \\
\text { y modos de empleo se ha } \\
\text { planteado en el modelo a } \\
\text { capital humano considera }\end{array}$ & $\begin{array}{l}\text { responsable de RRHH } \\
\text { vamente en el grado de } \\
\text { cticas utilizado por la } \\
\text { del modelo de Lepak y } \\
\text { por ser más o menos } \\
\text { de la FRH, las prácticas } \\
\text { ajustado conforme a lo } \\
\text { jalor y singularidad del } \\
\text { o. }\end{array}$ & $\begin{array}{l}\text { Existen ciertos ir } \\
\text { es el papel desen } \\
\text { menor es el nive } \\
\text { mayor homogen } \\
\text { todos los emple } \\
\text { significativas en } \\
\text { por el rol del res }\end{array}$ & $\begin{array}{l}\text { cios de que cuanto más estratégico } \\
\text { ñado por el responsable de la FRH, } \\
\text { ajuste al modelo, es decir } \\
\text { ad en las prácticas utilizadas con } \\
\text { os. No se evidencian diferencias } \\
\text { os diversos colectivos permeados } \\
\text { asable de RRHH. }\end{array}$ \\
\hline
\end{tabular}

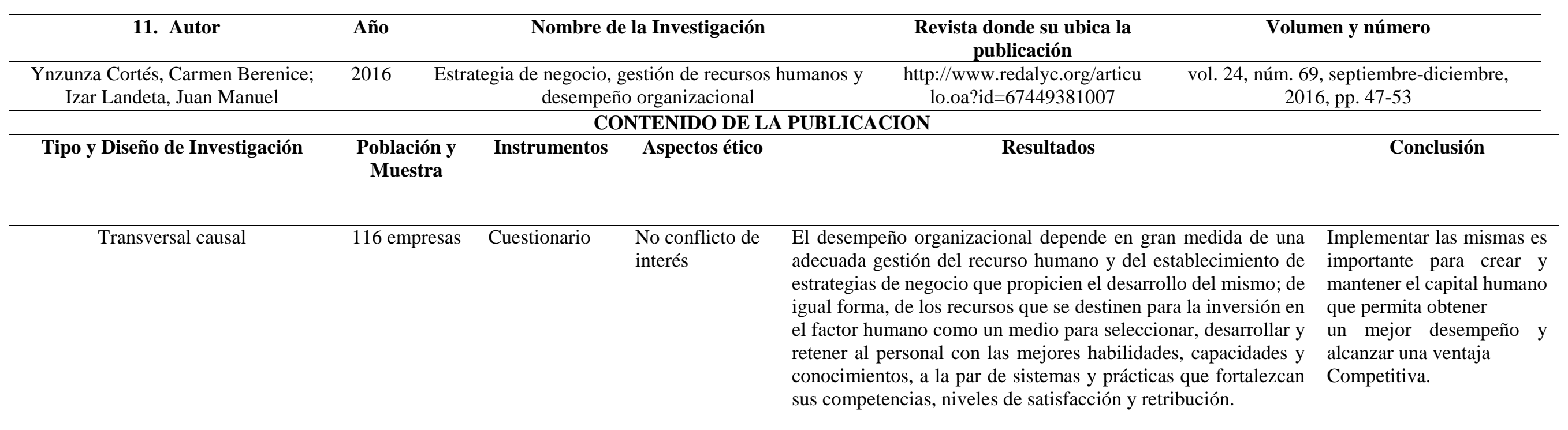

Ciencia Latina Revista Científica Multidisciplinar, Ciudad de México, México. 


\begin{tabular}{|c|c|c|c|c|c|c|c|}
\hline 12. Autor & Año & \multicolumn{4}{|c|}{$\begin{array}{c}\text { Nombre de la Investigación } \\
\end{array}$} & Revista donde su ubica la publicación & Volumen y número \\
\hline $\begin{array}{l}\text { Montoya Agudelo, } \\
\text { César Alveiro; }\end{array}$ & 2016 & \multicolumn{4}{|c|}{$\begin{array}{l}\text { El recurso humano como elemento fundamental para la gestión de } \\
\text { calidad y la competitividad organizacional }\end{array}$} & $\begin{array}{l}\text { http://www.redalyc.org/articulo.oa?id=3 } \\
\text { 57947335001 }\end{array}$ & $\begin{array}{l}\text { vol. 20, núm. } 2 \text {, julio-diciembre, } \\
\text { 2016, pp. } 1-20\end{array}$ \\
\hline \multicolumn{8}{|c|}{ CONTENIDO DE LA PUBLICACION } \\
\hline $\begin{array}{c}\text { Tipo y Diseño de } \\
\text { Investigación }\end{array}$ & & $\begin{array}{l}\text { ción y } \\
\text { estra }\end{array}$ & Instrumentos & Aspectos ético & & Resultados & Conclusión \\
\hline $\begin{array}{c}\text { Básico, descriptivo. } \\
\text { Revisión } \\
\text { documentaria }\end{array}$ & 90 & npañías & $\begin{array}{l}\text { Ficha de recojo de } \\
\text { datos }\end{array}$ & $\begin{array}{l}\text { No conflicto de } \\
\text { interés }\end{array}$ & $\begin{array}{l}\text { Necesidad de } \\
\text { una más vis } \\
\text { vinculándolo } \\
\text { direccionami } \\
\text { organizacion } \\
\text { capacidad de } \\
\text { consolidaciór } \\
\text { que el futur } \\
\text { capital humal }\end{array}$ & $\begin{array}{ll}\text { que la organización se oriente hacia } & \text { Reaf } \\
\text { on más ambiciosa de los RRHH, herr } \\
\text { como elementos claves del } & \text { los p } \\
\text { nto estratégico para el exito en } & \text { en u } \\
\text { l. El personal es el factor clave en la } & \text { la or } \\
\text { daptación de la organización y en la } & \text { para } \\
\text { de una ventaja competitiva, de ahí la ca } & \text { la aquella dependa de lograr un } \\
\text { de gara } \\
\text { o idóneo y comprometido. }\end{array}$ & $\begin{array}{l}\text { ma la necesidad de consolidar } \\
\text { mientas ropendan por la calidad de } \\
\text { ocesos, los productos y los servicios } \\
\text { sistema de calidad, de forma tal que } \\
\text { anización esté sustentada desde los } \\
\text { igmas del mejoramiento continuo y } \\
\text { didad total como dimensiones que le } \\
\text { tizan su viabilidad. }\end{array}$ \\
\hline
\end{tabular}

\begin{tabular}{|c|c|c|c|c|c|}
\hline 13. Autor & Año & \multicolumn{2}{|c|}{ Nombre de la Investigación } & Revista donde su ubica la publicación & $\begin{array}{l}\text { Volumen y número } \\
\end{array}$ \\
\hline $\begin{array}{l}\text { Paredes León, Karelly } \\
\text { C. }\end{array}$ & 2015 & \multicolumn{2}{|c|}{ El profesional de recursos humano } & $\begin{array}{c}\text { nos } \\
\text { http://www.redalyc.org/articulo.oa?id=4 } \\
65545899001\end{array}$ & $\begin{array}{l}\text { núm. 2, julio-diciembre, 2015, pp. } \\
\text { 265-268 }\end{array}$ \\
\hline \multicolumn{6}{|c|}{ CONTENIDO DE LA PUBLICACION } \\
\hline $\begin{array}{l}\text { Tipo y Diseño de } \\
\text { Investigación }\end{array}$ & $\begin{array}{c}\begin{array}{c}\text { Población y } \\
\text { Muestra }\end{array} \\
\end{array}$ & Instrumentos & $\begin{array}{c}\text { Aspectos } \\
\text { ético }\end{array}$ & Resultados & Conclusión \\
\hline Básico, descriptivo. & 96 profesionales & $\begin{array}{l}\text { Ficha de recojo de } \\
\text { datos }\end{array}$ & $\begin{array}{l}\text { No conflicto } \\
\text { de interés }\end{array}$ & $\begin{array}{l}\text { Dentro de la perspectiva de los múltiples roles } \\
\text { del recurso humano (estratégico, agente de } \\
\text { cambio, experto y adalid); con temas que tratan } \\
\text { sobre la salud y seguridad en el teletrabajo, la } \\
\text { aplicación de la teoría de los subconjuntos } \\
\text { borrosos en la gestión de personal, la gestión de } \\
\text { perfiles de cargos por competencia bajo un } \\
\text { enfoque ontológico. }\end{array}$ & $\begin{array}{l}\text { ancia que tiene para todo líder } \\
\text { múltiples roles que le corresponde } \\
\text { ndo de gestión de personas se trata. }\end{array}$ \\
\hline
\end{tabular}

Ciencia Latina Revista Científica Multidisciplinar, Ciudad de México, México. ISSN 2707-2207 / ISSN 2707-2215 (en línea), julio-diciembre, 2020, Volumen 4, Número 2. https://doi.org/10.37811/cl_rcm.v4i2.149 p. 1214 


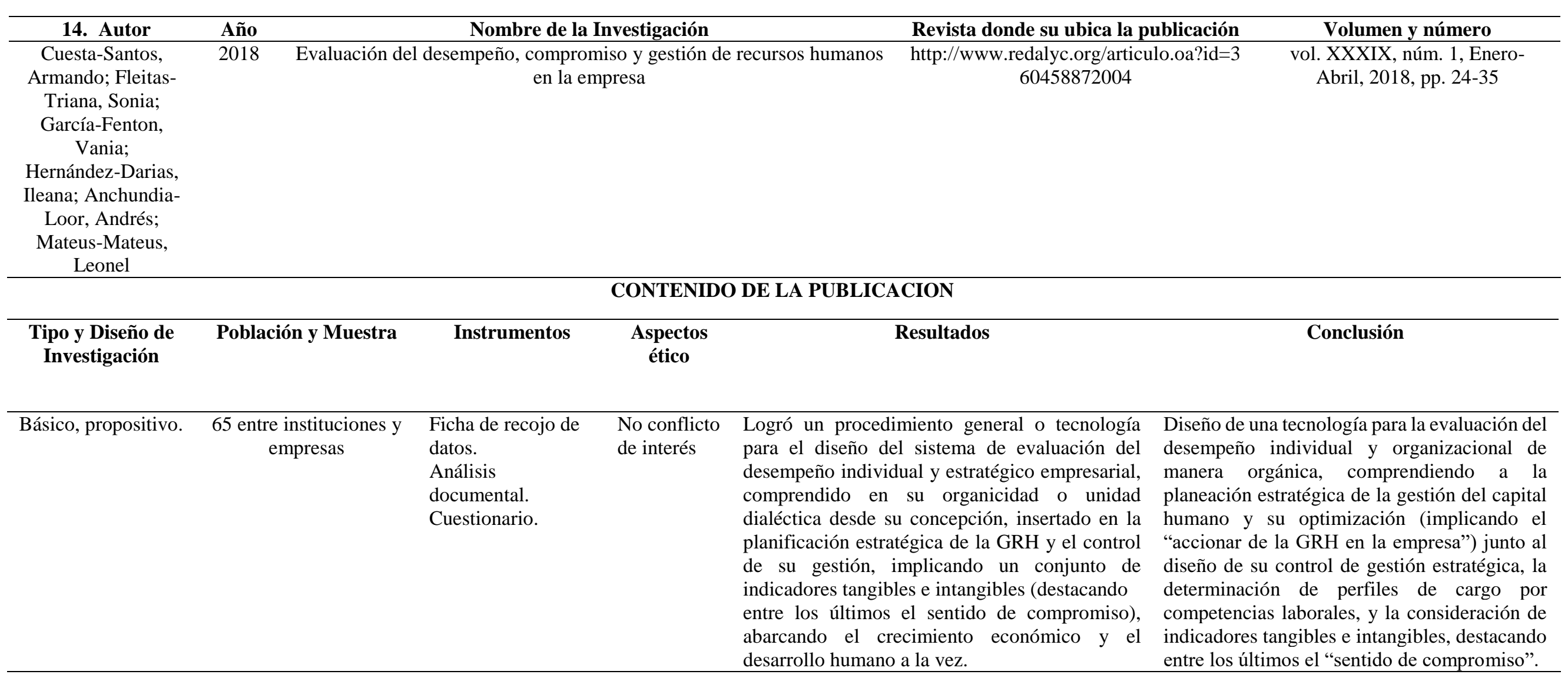

\begin{tabular}{ccccc}
\hline 15. Autor & Año & Nombre de la Investigación & Revista donde su ubica la publicación & Volumen y número \\
\hline Muñoz, José Luís; & 2015 & $\begin{array}{c}\text { Protagonismo de los Municipios en el Desarrollo Educativo: Propuesta } \\
\text { Gairín, Joaquín }\end{array}$ & de un Modelo e Instrumento para su Análisis & vol. 13, núm. 2, 2015, pp. 147- \\
\hline
\end{tabular}

Ciencia Latina Revista Científica Multidisciplinar, Ciudad de México, México.

ISSN 2707-2207 / ISSN 2707-2215 (en línea), julio-diciembre, 2020, Volumen 4, Número 2. https://doi.org/10.37811/cl rcm.v4i2.149 p. 1215 


\section{CONTENIDO DE LA PUBLICACION}

\begin{tabular}{|c|c|c|c|c|c|}
\hline $\begin{array}{l}\text { Tipo y Diseño de } \\
\text { Investigación }\end{array}$ & Población y Muestra & Instrumentos & $\begin{array}{c}\text { Aspectos } \\
\text { ético }\end{array}$ & Resultados & Conclusión \\
\hline $\begin{array}{l}\text { Descriptivo, } \\
\text { propositivo. }\end{array}$ & 17 participantes & Entrevista & $\begin{array}{l}\text { No conflicto } \\
\text { de interés }\end{array}$ & $\begin{array}{l}\text { Los Ayuntamientos, que representan la } \\
\text { administración más próxima a la ciudadanía, han } \\
\text { de aprovechar sus fortalezas relacionadas con el } \\
\text { mejor conocimiento de la realidad y las } \\
\text { necesidades educativas y ponerlas al servicio del } \\
\text { desarrollo educativo. Disponen de oportunidades } \\
\text { para implicarse en el desarrollo educativo como } \\
\text { consecuencia de un poder municipal emergente, } \\
\text { la tendencia a gestionar con mayor cercanía, el } \\
\text { prestigio de los servicios educativos que prestan } \\
\text { y la capacidad para promover el debate y la } \\
\text { innovación en educación. }\end{array}$ & $\begin{array}{l}\text { Remarcan la importancia que tienen } \\
\text { la confianza, el diálogo y el consenso para } \\
\text { mejorar la implicación de los municipios } \\
\text { en la educación; asimismo, la necesidad y la } \\
\text { conveniencia de que los Ayuntamientos } \\
\text { pongan en marcha políticas educativas amplias, } \\
\text { transversales e innovadoras para favorecer el } \\
\text { desarrollo educativo al que se aspira. }\end{array}$ \\
\hline
\end{tabular}

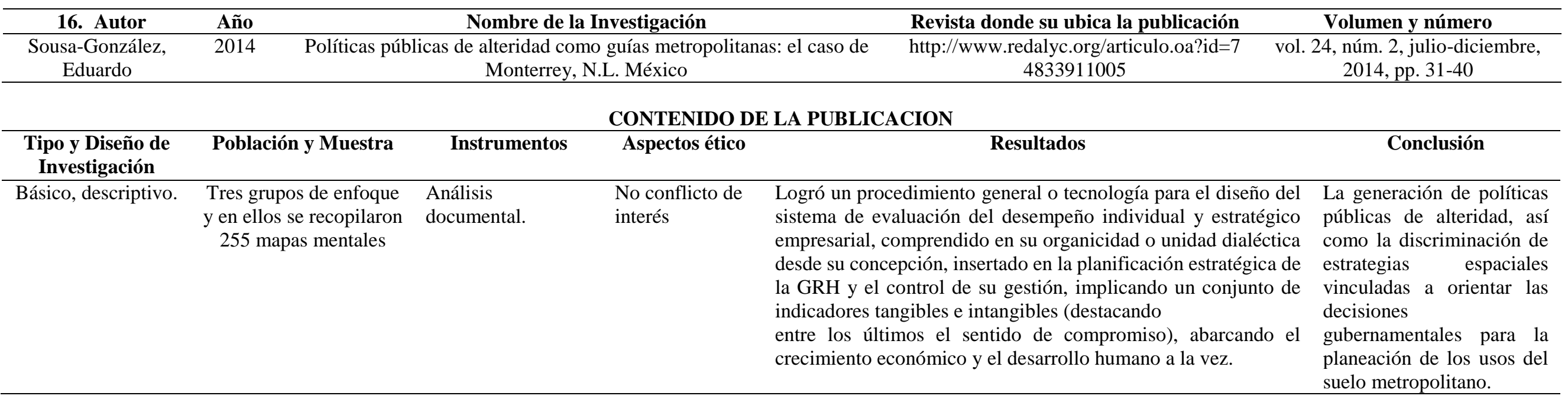

Ciencia Latina Revista Científica Multidisciplinar, Ciudad de México, México. 


\begin{tabular}{|c|c|c|c|c|c|c|}
\hline \multicolumn{5}{|c|}{ Nombre de la Investigación } & Revista donde su ubica la publicación & Volumen y número \\
\hline $\begin{array}{l}\text { Paprocki, Letizia } \\
\text { Mariel }\end{array}$ & \multicolumn{4}{|c|}{$\begin{array}{l}\text { Análisis de las variables para la valoración de la transparencia en la } \\
\text { administración de municipios }\end{array}$} & $\begin{array}{l}\text { http://www.redalyc.org/articulo.oa?id=3 } \\
57951171001\end{array}$ & $\begin{array}{l}\text { vol. 21, núm. 1, enero-junio, } \\
\text { 2017, pp. } 1-20\end{array}$ \\
\hline \multicolumn{7}{|c|}{ CONTENIDO DE LA PUBLICACION } \\
\hline $\begin{array}{l}\text { Tipo y Diseño de } \\
\text { Investigación }\end{array}$ & $\begin{array}{l}\text { Población y } \\
\text { Muestra }\end{array}$ & Instrumentos & Aspectos ético & & Resultados & Conclusión \\
\hline Básico, descriptivo. & $\begin{array}{l}\text { Municipalidades de } \\
\quad 3 \text { países }\end{array}$ & $\begin{array}{l}\text { Ficha de } \\
\text { recolección de } \\
\text { datos. }\end{array}$ & $\begin{array}{l}\text { No conflicto de } \\
\text { interés }\end{array}$ & $\begin{array}{l}\text { En función c } \\
\text { acciones o mo } \\
\text { adelante por } \\
\text { completo de } \\
\text { municipal, } \\
\text { fundamentale } \\
\text { a través de } \\
\text { transparencia }\end{array}$ & $\begin{array}{l}\text { cuatro experiencias concretas de promoción, } \\
\text { lelos de administraciones transparentes llevados } \\
\text { liferentes países, se ha definido un concepto } \\
\text { ansparencia que puede ser aplicado a la gestión } \\
\text { onteniendo este una serie de elementos } \\
\text { que luego son transformados en variables, que } \\
\text { indicadores permiten evaluar el grado de } \\
\text { le las gestiones. }\end{array}$ & $\begin{array}{l}\text { El análisis y la definición de } \\
\text { las variables para la } \\
\text { valoración de la } \\
\text { transparencia en la } \\
\text { administración municipal, } \\
\text { sus indicadores, y la } \\
\text { valoración e impactos de los } \\
\text { mismos, permite realizar un } \\
\text { avance para el estudio de la } \\
\text { transparencia de las gestiones } \\
\text { municipales, y así, sentar las } \\
\text { bases para la posterior } \\
\text { formulación de un modelo de } \\
\text { administración transparente } \\
\text { para municipios.. }\end{array}$ \\
\hline
\end{tabular}

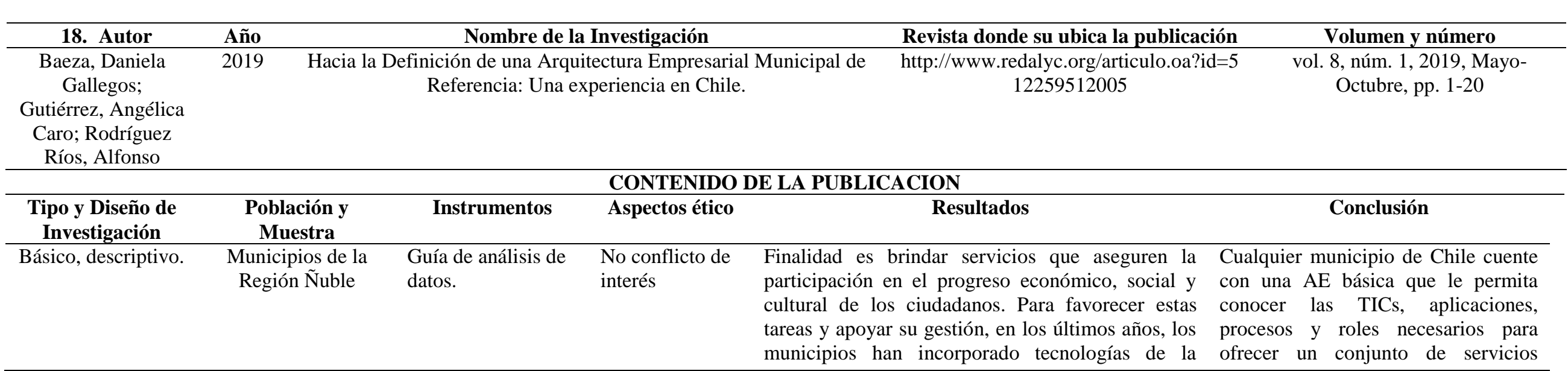

Ciencia Latina Revista Científica Multidisciplinar, Ciudad de México, México.

ISSN 2707-2207 / ISSN 2707-2215 (en línea), julio-diciembre, 2020, Volumen 4, Número 2. https://doi.org/10.37811/cl rcm.v4i2.149 p. 1217 
información y comunicación como una estrategia eficaz. mínimos a sus ciudadanos. Logrando con ello mayor equidad, de modo que independientemente del tamaño, ubicación y tipo de municipio los ciudadanos tengan acceso a los mismos servicios.

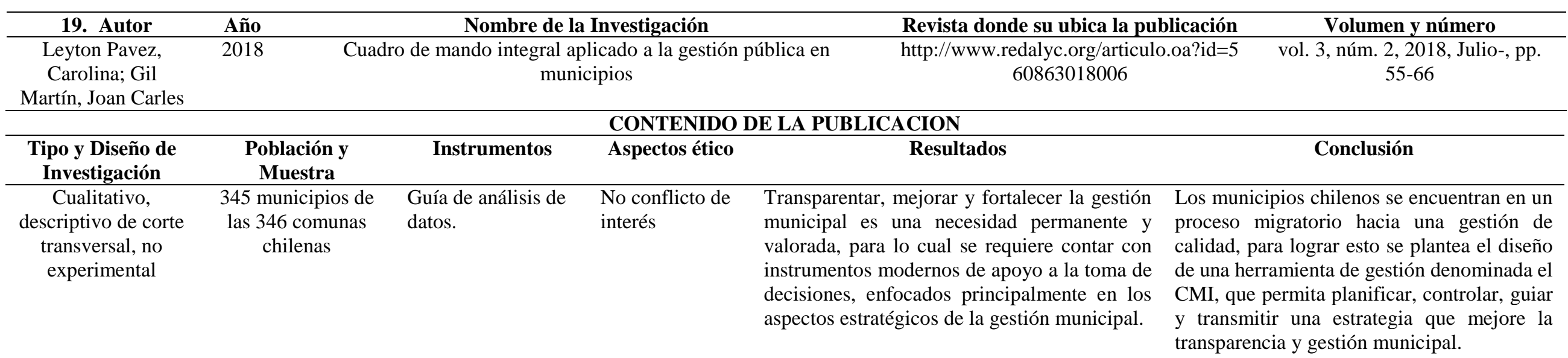

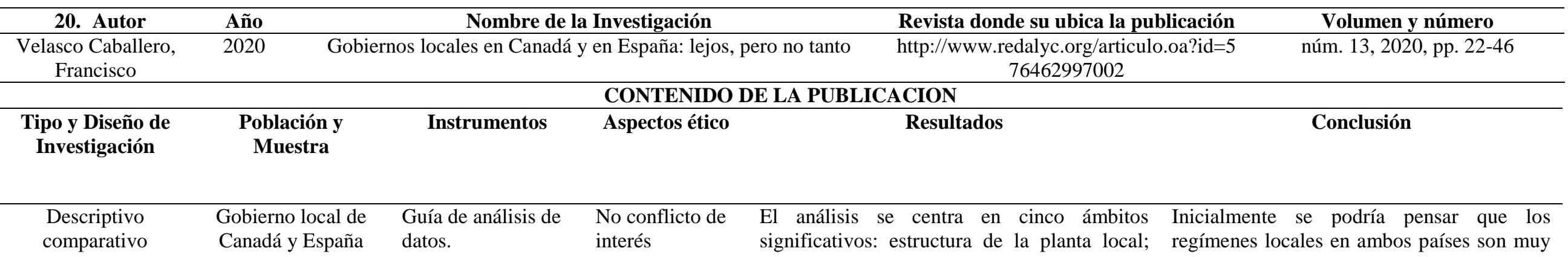

Ciencia Latina Revista Científica Multidisciplinar, Ciudad de México, México. 
autonomía; democracia; forma de gobierno; y financiación. distantes, visto con más profundidad hay tanto diferencias claras (en la planta local, las formas democráticas y la forma de gobierno) como también similitudes importantes: en el nivel de autonomía local real y en el sistema financiero.

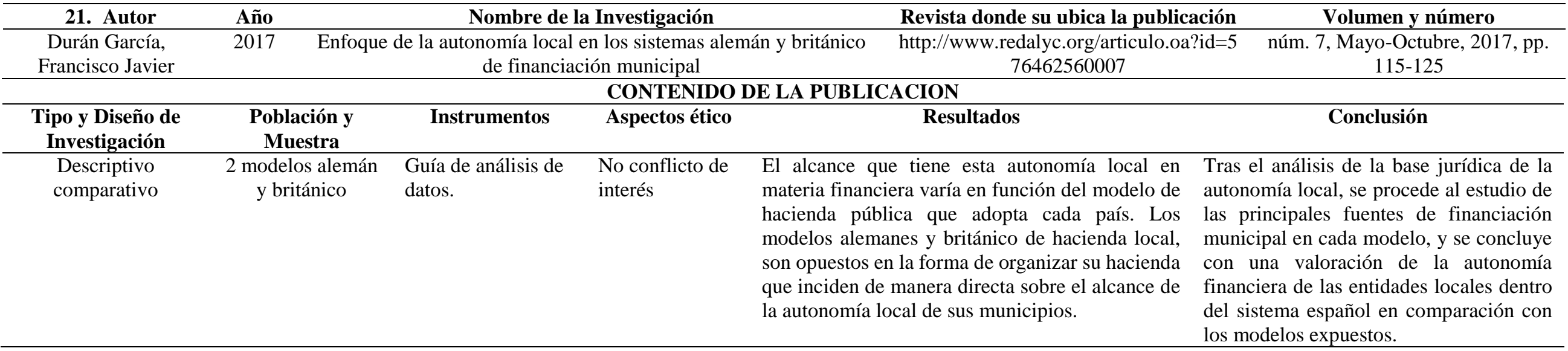

Ciencia Latina Revista Científica Multidisciplinar, Ciudad de México, México. 


\section{Discusión}

Los hallazgos encontrados en los estudios respecto a la gestión de recursos humanos, en el ámbito de América Latina, donde es considerada como una necesidad de contar con el recurso humano idóneo y comprometido, donde aportan con sus capacidades en el cumplimiento de objetivos y logros de metas por los gobiernos subnacionales, siendo este término el más empleado por el Banco Mundial; en ese sentido, el recurso humano es considerado como imput (insumo) en los diferentes procesos que desarrolla la gestión municipal donde no solamente articula procesos, si no también se relaciona con la ciudadanía, siendo capaz de escuchar y canalizar sus necesidades y convertirlo en la generación de valor público para la gestión edil. Debiendo ser garantizado con un perfil profesional académico de nivel superior para estar preparado y sensibilizado para comprender de mejor manera a sus semejantes, facilitando la interpretación de necesidades, donde además tiene que tener en su haber elementos y técnicas para la conducción del gobierno y la administración, abarcando el mayor número de estudios relacionados a esta temática.

De acuerdo a la temática abordada existen bastantes argumentos para coincidir con la afirmación de que podríamos estar ante la posibilidad de un colapso estructural, lo que habría que asumir como una oportunidad para una reforma de todo el Sistema de Servicios Sociales. En todo caso, hay que dejar de hacer una atribución externa de las responsabilidades y asumir las propias, que pasan, qué duda cabe, por un profundo cambio de paradigma que conduzca a la ineludible refundación de los Servicios Sociales municipales. En ese sentido, muchos estudios concluyeron que la estructura prestacional de servicios debe ser abordado para generar una nueva propuesta prestacional de servicio, mostrando la agilidad en poder responder las necesidades de los ciudadanos. Esta reforma estructural debe ser prioritario en dar soluciones emergentes, en donde pueda contextualizar a la realidad, en donde la oferta pueda alcanzar una mayor cobertura de las necesidades de los ciudadanos, con ello mejorar y alcanzar un mayor bienestar.

A ello se adiciona en la necesidad de apoyarse en la incorporación de la tecnología, así como su gestión, lo que conlleva a que los gobernantes, ciudadanía, empresas y demás actores involucrados en los municipios, propicien una visión enfocada a la gestión tecnológica en el municipio y hacer que los servicios puedan generarse de manera eficiente. 
Por otro lado, existe un grupo de investigadores, discrepan en la necesidad de consolidar herramientas que mejoren estandarizar elementos de calidad en los procesos, productos y en los servicios, de forma tal que la organización esté sustentada desde los paradigmas del mejoramiento continuo y la calidad total como dimensiones que le garantizan su viabilidad. Es así, que muchos autores se centran en mejorar procesos internos y externos de la gestión, otro grupo en insertar herramientas tecnológicas para agilizar la entrega de servicios, otro grupo se centra en el recurso humano para garantizar servicios oportunos y eficientes para los ciudadanos, donde incorpora el perfil y las capacidades de los prestadores de servicio, encabezado por el principal decisor que es el alcalde, donde su formación es determinante en las decisiones técnico político. Otro grupo se centra en propiciar una reestructuración de la organización, acercándose más en el ciudadano, respondiendo a sus demandas.

De acuerdo a lo mencionado es necesario contar con un gobierno municipal cercano a los ciudadanos, donde le permita identificar sus necesidades, siendo un elemento indispensable para ser considerado como prioridad en los documentos de gestión, para luego ser canalizados mediante la asignación presupuestal, donde incorpore el compromiso de los gestores públicos en el buen uso, debido a los escasos recursos y a las múltiples necesidades. Donde además, es necesario contar con procesos que acorten los procesos administrativos burocráticos que entorpecen la gestión, convirtiéndole muchas veces en generador de insatisfacción en los ciudadanos. Asimismo, es conveniente insertar herramientas tecnológicas, para hacer frente al contexto actual, donde es necesario acercarse a la ciudadanía mediante una plataforma, pudiendo realizar todo tramite desde un computador o smartphone, incrementando con ello la conectividad entre ciudadano - gobierno local.

\section{CONSIDERACIONES FINALES.}

Los tipos de estudios de mayor predominancia en la revisión sistemática son los básicos, con diseño descriptivo; a ello se adiciona los estudios comparativos y propositivos.

La revisión sistemática, se sustentó en la gestión de recursos humanos y la prestación de servicios; el primero evidencia que es una variable determinante para gestionar adecuadamente; la gestión local, tiene otras denominaciones como ayuntamiento, municipio, municipalidad, gobierno subnacional todos ellos buscan responder adecuadamente a la demanda poblacional, donde incorpora diferentes miradas, como 
social, económico, medio ambiental e institucional; todo ello depende de la circunstancias y la demanda de los ciudadanos en el territorio que gobierna.

Por su parte, la prestación de servicios debe tener características fiables para los ciudadanos, donde primero debe cerrar brechas, ampliando la cobertura de servicios, a ello agregar la calidad y cantidad del servicio; en busca de generar valor público que sea capaz de satisfacer sus necesidades y expectativas.

De la misma manera, los investigadores precisan que es pertinente mejorar las condiciones institucionales, principalmente la estructura y funcionalidad, que son elementos fundamentales para la reestructuración organizacional, donde facilitara el acercamiento a la ciudadanía.

\section{LISTA DE REFERENCIAS}

Aguinis, H., \& O'Boyle, E. (2014). Star performers in twenty-first century organizations. Personnel Psychology, 67(2), 313-350.

Becker, B., Huselid, M., \& Beatty, R. (2009). Differentiated Workforce. Transforming Talent into Strategic Impact. Boston: MA: Harvard Business School Press.

Bonardo, D. (2009). Los recursos humanos en el ámbito municipal y el desarrollo local. Pilquen, 1-7. Obtenido de http://www.redalyc.org/articulo.oa?id=347532055001 Boyero, M., \& Montoya, C. (2016). El recurso humano como elemento fundamental para la gestión de calidad y la competitividad organizacional. Visión Futura, 1-20. Obtenido de http://www.redalyc.org/articulo.oa?id=357947335001

Cappelli, P. (2000). A market-driven approach to retaining talent. Harvard Business Review, 78(1), 103-111.

Chaín, C., Muñoz, A., \& Más, A. (2008). La gestión de información en las sedes web de los ayuntamientos españoles. Revista española de documentación científica, 31, 612-638.

Cuesta-Santos, A., Fleitas-Triana, S., García-Fenton, V., Hernández-Darias, 1., Anchundia-Loor, A., \& Mateus-Mateus, L. (2018). Evaluación del desempeño, compromiso y gestión de recursos humanos en la empresa. Ingeniería Industrial, 24-25. Obtenido de http://www.redalyc.org/articulo.oa?id=360458872004

Delery, J., \& Doty, D. (1996). Modes of theorizing in Strategic Human Resource Management: Test of Universalistic, Contingency, and Configurational Performance Predictions. Academy of Management Journal, 39(4), 802-835. 
Durán, F. (2017). Enfoque de la autonomía local en los sistemas alemán y británico de financiación municipal. Revista de Estudios de la Administración Local y Autonómia, 115-125. doi:10.24965/reala.v0i7.10396

Fernández, S., Martín, F., \& Sánchez, G. (2015). Flexibilidad funcional en la dirección estratégica de los recursos humanos. Revista de Ciencias Sociales, 321-331. Obtenido de http://www.redalyc.org/articulo.oa?id=28042299004

Gallegos-Baeza, D., Caro, A., \& Rodríguez, A. (2019). Hacia la Definición de una Arquitectura Empresarial Municipal de Referencia: Una experiencia en Chile. Revista electrónica de Computación, Informática, Biomédica y Electrónica, 1-20. Obtenido de http://www.redalyc.org/articulo.oa?id=512259512005

Gónzalez, R. (2005). Creando valor con la gente. México: Norma.

Hernández, R. (2014). Metodología de la Investigación (Sexta ed.). México: Mc Graw Hill.

Hernández, R. (2016). Metodología de la Investigación. Lima: Mc Graw Hill.

Instituto Nacional de Administración Pública A.C. (INAP). (2009). Guías técnicasmunicipales. Ciudad de México: Instituto Nacional de Administración Pública

A.C. Obtenido

de http://www.inafed.gob.mx/work/models/inafed/Resource/322/1/images/guias_in ap.pdf

Leyton, C., \& Gil, J. (2018). Cuadro de mando integral aplicado a la gestión pública en municipios. Revista Academia \& Negocios, 55-66. Obtenido de http://www.redalyc.org/articulo.oa?id=560863018006

López-Cabanas, M. (2018). Servicios sociales municipales. Necesidad de una refundación. Papeles del Psicólogo, 2-15. Obtenido de http://www.redalyc.org/articulo.oa?id=77855949002

Medina, A., Revuelto, L., \& Acosta, A. (2017). Perfil del responsable y su impacto en la arquitectura de Recursos Humanos. Revista Vnezonla de Gerencia, 75-94. Obtenido de http://www.redalyc.org/articulo.oa?id=29051457006

Millán-Valenzuela, H., \& García-Pérez, M. (2017). Instituciones y desarrollo municipal: Un análisis multinivel. Economía Sociedad y Territorio, 63-86. Obtenido de http://www.redalyc.org/articulo.oa?id=11149081004 
Muñoz, J., \& Gairín, J. (2015). Protagonismo de los Municipios en el Desarrollo Educativo: Propuesta de un Modelo e Instrumento para su Análisis. Revista Iberoamericana sobre Calidad, Eficacia y Cambio en Educación, 147-161. Obtenido de http://www.redalyc.org/articulo.oa?id=55138743008

Olivos, J. (2013). Gobernación municipal en México: alcances y desafíos. Revista del Instituto de Ciencias Jurídicas, 118-147. Obtenido de http://www.redalyc.org/articulo.oa?id=293229863008

Ortíz, J., Rendón, M., \& Atehortúa, J. (2012). Score de competencias: cómo transformar el modelo de competencias de su empresa en un sistema de "Score" asociado a los proceso clave de su negocio. Madrid: Palibrio.

Paprocki, L. (2017). Análisis de las variables para la valoración de la transparencia en la administración de municipios. Visión Futuro, 1-20. Obtenido de http://www.redalyc.org/articulo.oa?id=357951171001

Paredes, K. (2015). El profesional de recursos humanos. Visión Gerencial, 265-268. Obtenido de http://www.redalyc.org/articulo.oa?id=465545899001

Pérez, A., \& Luján, D. (2018). Cercanía, favor, lealtad. Clientelismo en dos municipalidades chilenas. Sociológica, 235-268. Obtenido de http://www.redalyc.org/articulo.oa?id=305062917008

Pompilio, A. (2016). Comunicación política y gestión municipal. Espacios Públicos, 135155. Obtenido de http://www.redalyc.org/articulo.oa?id=67650281006

Rivero, A., \& Dabos, G. (2017). Gestión diferencial de recursos humanos: una revisión e integración de la literatura. Estudios gerenciales, 1-30. Obtenido de http://www.redalyc.org/articulo.oa?id=21252448005

Rubio-Núñez, R., Valencia, L., Peña-Cheng, L., \& Rodríguez-Muñoz, E. (2018). Importancia de la Gestión Tecnológica en los Gobiernos Municipales Mexicanos. Gestión de las Personas y Tecnología, 1-10. Obtenido de http://www.redalyc.org/articulo.oa?id=477857553008

Sá, F., Rocha, Á., \& Pérez, M. (2016). Potential dimensions for a local e-Government services quality model. Telematics and Informatics, 33(2), 270-276.

Sánchez, M., \& Jaimes, O. (2017). Profesionalización de políticos y funcionarios municipales. Espacios Públicos, 1-29. Obtenido de http://www.redalyc.org/articulo.oa?id=67656393004 
Sandoval-Almazan, R., \& Mendoza, J. (2011). Gobierno electrónico en México : una exploración Municipal 2010. In AMCIS SUBDERE. Portal de Servicios Municipales. Obtenido de https://www.sem.gob.cl/servicios.php.

Souza-González, E. (2014). Políticas públicas de alteridad como guías metropolitanas: el caso de Monterrey, N.L. México. Bitácora Urbano Territorial, 31-40. Obtenido de http://www.redalyc.org/articulo.oa?id=74833911005

Supervielle, M., \& Pucci, F. (2015). Procesos de trabajo, organización y gestión de los recursos humanos. Ciencias Sociales, 9-11. Obtenido de http://www.redalyc.org/articulo.oa?id=453644798001

Velasco, F. (2020). Gobiernos locales en Canadá y en España: lejos, pero no tanto. Revista de Estudios de la Administración Local y Autonómica, 22-46. doi:10.24965/reala.i13.10742

Wollmann, H. (2019). La provisión de servicios públicos municipales: ¿un movimiento pendular? $\quad$ Barataria, 15-29. doi:DOI: https://dx.doi.org/10.20932/barataria.v0i26.457

Ynzunza, C., \& Izar, J. (2016). Estrategia de negocio, gestión de recursos humanos y desempeño organizacional. Investigación y Ciencia, 47-53. Obtenido de http://www.redalyc.org/articulo.oa?id=67449381007 